\title{
EFFECT OF A CLUSTER ON GAS-SOLID DRAG FROM LATTICE BOLTZMANN SIMULATIONS
}

\author{
$\underline{\text { Milinkumar T. Shah }}{ }^{1}$, Ranjeet P. Utikar ${ }^{1,{ }^{*}}$, Moses O. Tade ${ }^{1}$, Geoffrey M. Evans $^{2}$ and Vishnu K. Pareek ${ }^{1}$ \\ ${ }^{1}$ Department of Chemical Engineering, Curtin University, Perth, WA 6845, AUSTRALIA \\ ${ }^{2}$ Department of Chemical Engineering, University of Newcastle, NSW 2308, AUSTRALIA \\ *Corresponding author, E-mail address: r.utikar@curtin.edu.au
}

\begin{abstract}
Formation of particle clusters in fast fluidization of fine particles significantly affects the gas-solid drag force. Accounting for the effect of clusters in gas-solid drag is critical for accurate modelling of gas-solid flows. As a result several modifications to the empirical gas-solid drag models have been proposed. However, computational studies with these modified drag models have shown their limitations in capturing the inherent heterogeneity found in the gas-sold flow. Generally, this has been attributed to the lack of understanding on effects of cluster on the gassolid drag force. In this study, direct numerical simulations using lattice Boltzmann method have been conducted to investigate the effect a single cluster and its properties such as cluster voidage and fraction on the gas-solid drag force over a wide range of overall voidages and particle Reynolds numbers. The numerical observations clearly show that particle configuration with a cluster exhibit a considerably lower drag than particles in random arrangement. Furthermore, major drag reduction is observed when the inter-particle distances within the cluster decreases for voidage ranging from maximum voidage to 0.7 . The simulations show that for constant cluster voidage, minimum drag force occurred around 0.9 to 0.95 overall voidage. The drag force increased steeply with decrease in the overall voidage. The findings reported here will pave the way to improved drag correlation that can be used in CFD simulations that solve the average two-fluid equations.
\end{abstract}

\section{NOMENCLATURE}

$e_{a}$ Direction vector

$F \quad$ Momentum $(\mathrm{kg} \mathrm{m} / \mathrm{s})$

$F_{d}$ Drag force $\left(\mathrm{kg} \mathrm{m} / \mathrm{s}^{2}\right)$

$f$ Cluster fraction

$f_{a} \quad$ Velocity distribution function

$M \quad$ Mass (kg)

$r_{b} \quad$ Location of boundary node [lattice unit(lu)]

$R \quad$ Location of centre of mass of a particle (lu)

$T$ Torque $\left(\mathrm{kg} \mathrm{m}^{2} / \mathrm{s}^{2}\right)$

$t$ Time (s)

$\Delta t \quad$ Time step (s)

$U$ Velocity of particle (lu/s)

$u$ macroscopic velocity $(\mathrm{lu} / \mathrm{s})$

$u_{b} \quad$ Velocity at boundary node $(\mathrm{lu} / \mathrm{s})$ $x \quad$ Lattice node

$v$ Kinematic viscosity

$w \quad$ Weight functions

Greek Lettter

$\varepsilon \quad$ Voidage

$\rho \quad$ Macroscopic Density $\left(\mathrm{kg} / \mathrm{m}^{3}\right)$

$\rho_{w} \quad$ Density at wall

$\tau \quad$ Relaxation time (s)

$\mu \quad$ Viscosity $(\mathrm{kg} / \mathrm{m} \mathrm{s})$

$\pi \quad$ Macroscopic stress

$\Omega$ Angular velocity of particle

Subscript

a Direction

$b$ Boundary

c Cluster

d Drag

eq Equilibrium

$f$ fluid

$f-p$ Exchange between fluid and particle

$f-s$ Exchange between fluid and solid

$p$ Particle

\section{INTRODUCTION}

Gas-solid flow under fast fluidization conditions finds wide applications in chemical processes such as fluid catalytic cracking, circulating fluidized bed combustion, etc. The performance of these processes largely depends on hydrodynamics of gas-solid flow. Therefore, computational fluid dynamics (CFD) based gas-solid flow models have extensively been applied to investigate the hydrodynamics and possible design improvements. Such CFD models include mass and momentum conservations for both gas and solid phases along with inter-phase exchange gas-solid drag model, which is the most critical and dominant closure model. Most commonly used empirical gas-solid drag models such as (i) the Ergun model (Ergun, 1952), derived from pressure drop data under packed bed conditions; (ii) the Wen-Yu model (Wen and $\mathrm{Yu}, 1966)$, derived from single particle settling experiments; and (iii) the Gidaspow drag model (Gidaspow, 1994), a combination of the Ergun and Wen$\mathrm{Yu}$ model give reasonable predictions for drag force for voidages at two extremes i.e. maximum voidage and minimum fluidizing voidage. However none of these models account for the effect of formation of particle 
aggregates, so called clusters, which occurs at intermediate voidages.

To account for the effect of clusters, the conventional drag models have been modified using multi-scale approaches such as sub-grid scale (Andrews IV et al., 2005) and energy minimization approaches ( $\mathrm{Li}$ and Kawauk, 1994). Even with these modifications, the performances of CFD models have been limited to qualitative agreement. Quantitative validations have not been achieved yet (Benyahia, 2009; Shah et al., 2011b). Improvements in the current drag models are key to accurate predictions, and require better understanding of the effect of clusters. Available multiphase experimental techniques such as magnetic resonance imaging, computer tomography and radioactive particle tracking are ineffective in capturing the spatio-temporal scales required to analyse the gas-solid interactions at the scale of clusters. On the other hand, direct numerical simulations (DNS) of gas-particle flow can provide observations at a resolution as high as $10-20$ times less than particle diameter.

The Lattice Boltzmann method (LBM) has been previously used to simulate gas-solid flows in order to study interactions between two phases. Hill et al. (2001a, b) used the LBM to study the drag force on spheres, and provided first numerical observations which showed that the gas-solid drag over a range of solid volume fractions was different from that calculated using conventional drag models. However, their simulations were limited only to low particle Reynolds numbers and mono-dispersed randomly or regularly arranged particles. Van der Hoef et al. (2005) conducted LB simulations of fluid flowing past mono- and bi-disperse random arrays of spheres to measure the drag force on the spheres for a range of diameter ratios, mass fractions and packing fractions. From their numerical data, they gave a correlation for the drag force applicable to both mono- and poly-disperse systems. Beetstra et al. (2006) simulated particles arranged in a cluster that had different shapes. The numerically calculated drag coefficients were compared with the experimental data of drag coefficients for irregularly shaped particles reported by Tran-Cong et al. (2004). Beetstra et al. (2006) concluded a strong effect of interparticle distance on the gas-solid drag force. However, this study was limited to number of the particles as high as only 32 particles, and did not include the effect of particle Reynolds number on the drag force. Recently, Zhang et al. (2011) simulated a 2D periodic array of clusters using LBM to investigate the effect of cluster on the drag coefficient. They found close agreement between the simulated drag values and those calculated from the energy minimization approach.

While detailed gas-solid flow simulations are available, the effect of clusters on the gas-solid drag is still poorly understood. The present study aims to quantify the effect of cluster on gas-solid drag. 3D LB simulations of gassolid flow have been carried out to investigate the effect of a single cluster and its properties on the gas-solid drag force. The flow over different particle clusters (of up to 1331 particles) with various cluster diameters and cluster voidages have been studied under different flow conditions.

\section{LATTICE BOLTZMANN METHOD}

This section is intended to give a brief introduction to modelling multiphase flow using LBM. For detail understanding of the LBM, the readers are referred to Ladd (1994a and b). LBM is a direct numerical simulation technique which resolves the flow of fluid by solving the Boltzmann equation of velocity distributions and the movement of particles by solving Newton's force balance equation for each particle. The momentum exchange between the fluid and particles is resolved by applying the stick boundary condition, which says that the velocity of the fluid adjacent to a particle surface is equal to the local velocity of the surface at that point.

Flow of fluid: A flow domain is discretized with number of lattices in $\mathrm{x}, \mathrm{y}$ and $\mathrm{z}$ directions. Each node represents a fluid element with its velocity distributed in 19 directions. These fluid elements propagate from one grid point to the next at each time step, and the propagation includes two parts namely i.e. (i) streaming - movement from one grid point to another; and (ii) collision - collision between fluid elements coming at the same lattice node. The velocity distribution of a fluid element at each node is updated by the Boltzmann equation:

$f_{a}\left(x+e_{a}, t+\Delta t\right)=f_{a}(x, t)-\frac{\left[f_{a}(x, t)-f_{a}^{e q}(x, t)\right]}{\tau}$

where $f_{a}$ is velocity distribution at any lattice node $x, e_{a}$ is the direction vectors and $\tau$ is a relaxation time. Equation(1) has two parts, whree (i) $f_{a}\left(x+e_{a}, t+\Delta t\right)=$ $f_{a}(x, t)$ represents the streaming; and (ii) $\left[f_{a}(x, t)-\right.$ $\left.f_{a}^{e q}(x, t)\right] / \tau$ represents collision steps. Equation(1) represents the Bhatnagar-Gross-Krook (BGK) approach (Bhatnagar et al., 1954) for a relaxation towards equilibrium; where the relaxation time, $\tau$ is governed by the kinematic viscosity of the fluid, with length being represented in terms of lattice units (lu), i.e.;

$\tau=3 v+0.5$

Macroscopic properties such as density, velocity and stress are calculated from the velocity distribution functions at each lattice node using the following equations,

$\rho=\sum_{a=0}^{19} f_{a}$

$u=\sum_{a=0}^{19} f_{a} e_{a}$

$\pi=\sum_{a=0}^{19} f_{a} e_{a} e_{a}$

When a fluid element encounters a solid boundary, the bounce back rule is applied, i.e.:

$f_{-a}(x, t+\Delta t)=f_{a}(x, t)$

The macroscopic properties are used to calculate equilibrium distribution:

$f_{a}^{e q}=w^{e_{a}} \rho\left[1+\frac{u e_{a}}{c_{s}^{2}}+\frac{\left(u e_{a}\right)^{2}}{2 c_{s}^{4}}-\frac{u^{2}}{2 c_{s}^{2}}\right]$

where $c_{s}$ is the speed of sound and $w^{e a}$ is the weight function for different directions. The value of $c_{s}$ is $1 / 3$ lattice unit per second, whilstthose of $w^{0}, w^{1}$ and $w^{\sqrt{2}}$ are $1 / 3,1 / 18$ and $1 / 36$ respectively.

Fluid-particle interactions: If a fluid element strikes a moving solid boundary, such as suspended particles, then a stick boundary condition is applied and the velocities of the fluid elements at nodes inside the solid particle are equal to the velocity of the particle. The bounce back rule at the moving solid boundary is:

$f_{-a}(x, t+\Delta t)=f_{a}(x, t)-6 \rho_{w} w^{e_{a}}\left(u_{b} e_{a}\right)$ 
where $\rho_{w}$ is wall density, and $u_{b}$ is the velocity of the boundary nodes inside the particle. The value of $u_{b}$ is resultant of the axial and radial velocity of the particle, given by:

$u_{b}=U+\Omega \times\left(r_{b}-R\right)$

where $U$ is translation velocity of the particle, $\Omega$ is angular velocity, $r_{b}$ is location of boundary node and $R$ is the location of centre of mass of the particle.

The resulting force exerted by the fluid element on the particle because of the change in momentum of the fluid element is given by:

$F_{f-p}=\sum_{b} \sum_{a}\left[2 f_{a}(x, t)-6 \rho_{w} w_{a}\left(u_{b} e_{a}\right)\right] e_{a} ;$

whilst torque is given by:

$T_{f-p}=\sum_{b}\left(r_{b}-R\right) \sum_{a}\left[2 f_{a}(x, t)-6 \rho_{w} w_{a}\left(u_{b} e_{a}\right)\right] e_{a}$

Movement of particles: Newton's force balance equation is solved for each particle to obtain its velocity:

$F_{p}=M_{p} \frac{d}{d t} U$

$T_{p}=I_{p} \frac{d}{d t} \Omega$

where $F_{P}$ and $T_{P}$ are momentum and torque transfer to particles from fluid plus other forces. $M_{p}$ and $I_{p}$ are mass and moment of inertial of the particle.

The change in fluid momentum (eq. 8) at the boundary nodes on a single particle is equal and opposite of the total force that the gas exerts, $\mathrm{F}_{\mathrm{f}-\mathrm{s}}$. Using this equality, the drag force $F_{d}$ can be calculated.

\section{LB SIMULATIONS}

For the present study, a multiphase 3D lattice Botzmann code "SUSP3D" (Ladd, 1994a and b) was used. SUSP3D is a highly parallel code and scales well for thousands of particles. However, the size of the flow domain and number of particles in simulations are limited by the available computational power. For example, each simulation with a $256 \mathrm{lu}^{3}$ required more than $16 \mathrm{~GB}$ of RAM and 2.4 GB data storage capacity. Further increase in the size of the flow domain increases the demand on RAM and disc storage exponentially. Therefore, for the present study, the domain was limited to 256 lattices in each direction. The particle diameter was set at $17.5 \mathrm{lu}$ to keep the domain size to particle diameter ratio less than 20 , which is generally a cell size in continuum gas-solid flow simulations.

Simulations with random particle arrangement and with cluster configuration were performed. A list of the simulations conditions are given in Table-1.

Table 1: Simulation Conditions

\begin{tabular}{|c|c|c|}
\hline & $\begin{array}{l}\text { Initial } \\
\text { simulations } \\
\text { (Beetstra, 2005) }\end{array}$ & $\begin{array}{l}\text { Simulations } \\
\text { With cluster } \\
\text { configurations } \\
\end{array}$ \\
\hline Flow domain & Variable & 256 Lattice $^{3}$ \\
\hline Particle diameter & 17.5 Lattices & 17.5 Lattices \\
\hline Kinematic viscosity & $8.333 \mathrm{e}-4 \mathrm{lbu}$ & $8.333 \mathrm{e}-4 \mathrm{lbu}$ \\
\hline Fluid density & $36 \mathrm{lbu}$ & 36 LB lbu \\
\hline $\begin{array}{l}\text { Ratio of particle to } \\
\text { fluid density }\end{array}$ & 1 & 2000 \\
\hline Boundary condition & Periodic & Periodic \\
\hline Particle arrangement & Random & $\begin{array}{l}\text { A single cluster } \\
\text { with surrounding }\end{array}$ \\
\hline
\end{tabular}

$\begin{array}{lll}\text { Number of particle } & 54 & \text { particles } \\ \text { Number of time steps } & 1000 & \text { Variable } \\ \text { Number of cycles } & 100-150 & 1000 \\ \end{array}$

For simulations with random particle arrangement, configurations similar to Beetstra (2005) were chosen. The primary aim was to verify the LB simulation methodology used in this study. In these simulations, 54 particles were randomly positioned using the Monte-Carlo method in a cubical flow domain. The diameter of each particle was 17.5 lattices, and the size of the flow domain was adjusted to achieve a desired volume fraction. The simulations were set up in such a way that a particle configuration was forced to move with a velocity in x-direction. As a result the moving particle configuration exerted the drag force to the gas phase, which was counter balanced by applying a uniform force to the gas phase. This condition ensured that the particles moved as a static configuration, and their velocity was equal to superficial gas velocity. Thus, the particle Reynolds number was given by:

$N_{R e, p}=\rho U d / \mu=\rho V^{s i m} d / \mu$.

where $U$ is superficial gas velocity, $V_{\text {sim }}$ is velocity of particles. Variation in particle Reynolds number was obtained by changing the flow velocity. The volume fractions, domain sizes and particle Reynolds numbers used in the simulations are listed in Table- 2 .

Table 2: Initial simulation runs of Beetstra, 2005

\begin{tabular}{ll|ll}
\hline $\begin{array}{l}\text { Vol. } \\
\text { fraction }\end{array}$ & $\begin{array}{l}\text { Flow domain } \\
\left(\mathrm{lu}^{3}\right)\end{array}$ & $\begin{array}{l}\text { Particle Reynolds } \\
\text { Number }\left(\mathrm{N}_{\mathrm{Re}, \mathrm{p}}\right)\end{array}$ & $\begin{array}{l}\text { Velocity } \\
\left(\mathrm{lu} \mathrm{s} \mathrm{s}^{-1)}\right.\end{array}$ \\
\hline 0.1 & 120 & 0.2 & $1 \times 10^{-5}$ \\
0.2 & 92 & 21 & $1 \times 10^{-3}$ \\
0.3 & 81 & 105 & $5 \times 10^{-3}$ \\
0.4 & 74 & 210 & $1 \times 10^{-2}$ \\
0.5 & 69 & 420 & $2 \times 10^{-2}$ \\
& & 1049 & $5 \times 10^{-2}$ \\
\hline
\end{tabular}

The simulations were run in a periodic domain for 150 cycles. The first 100 cycles were required to achieve a steady state, whilst the last 50 cycles were used for averaging. The force exerted on each particle obtained by simulation was divided by voidage to calculate the gasparticle drag force. The calculated drag force was normalized by the Stokes-Einstein drag force on a single particle under zero Reynolds number limit (Hill et al., $2001 \mathrm{~b}$ and Beetstra et al., 2005) The averaged normalized drag force is reported. The relevant equations are:

$F_{f-s}=\frac{F_{d}}{\varepsilon}$

$F_{f-s, \text { normalized }}=\frac{F_{f-s}}{F_{d, s t}}=\frac{F_{f-s}}{3 \pi \mu d U}$

where $F_{f-s}$ is total drag force, $F_{d}$ is drag force on a single particle, $F_{f-s, \text { normalized }}$ is the dimentionless normalized drag force, $F_{d, s t}$ is the Stokes-Einstein drag, $\mu$ is viscosity of fluid, $d$ is diameter of particle and $U$ is superficial velocity of fluid.

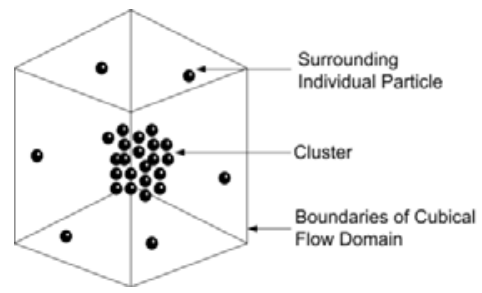


Figure 1: Particle configurations used in each simulation.

For simulations with cluster configurations, particles were positioned in cluster configurations with each consisted of a single cluster of particles and several surrounding individual particles as shown in Figure-1. Details of particle configurations are summarized in Table- 3 .

Table 3: Details of Particle Configurations

\begin{tabular}{lllllll}
\hline & particles & $\begin{array}{l}\text { particles } \\
\text { in cluster }\end{array}$ & $\begin{array}{l}\text { particles in } \\
\text { surrounding }\end{array}$ & $\begin{array}{l}\text { Cluster } \\
\text { voidage }\end{array}$ & $\begin{array}{l}\text { Overall } \\
\text { voidage }\end{array}$ & $\begin{array}{l}\text { Cluster } \\
\text { fraction }\end{array}$ \\
\hline 1 & 90 & 64 & 26 & 0.9 & 0.9850 & 0.1505 \\
2 & 90 & 64 & 26 & 0.8 & 0.9850 & 0.0752 \\
3 & 90 & 64 & 26 & 0.7 & 0.9850 & 0.0502 \\
4 & 90 & 64 & 26 & 0.6 & 0.9850 & 0.0376 \\
5 & 90 & 64 & 26 & 0.52 & 0.9850 & 0.0313 \\
6 & 151 & 125 & 26 & 0.7 & 0.9748 & 0.0841 \\
7 & 242 & 216 & 26 & 0.7 & 0.9595 & 0.1349 \\
8 & 538 & 512 & 26 & 0.7 & 0.9101 & 0.2998 \\
9 & 1026 & 1000 & 26 & 0.7 & 0.8285 & 0.5717 \\
10 & 1331 & 1331 & 0 & 0.7 & 0.7775 & 0.7417 \\
\hline
\end{tabular}

Various voidages of the particle configurations listed in Table- 3 can be correlated by:

$\varepsilon=(1-f) \varepsilon_{f}+f \varepsilon_{c}$

where $\varepsilon$ is overall voidage, $f$ is cluster fraction, $\varepsilon_{f}$ is voidage of surrounding fluid and $\varepsilon_{c}$ is cluster voidage. Note that due to small number of particles present, the voidage of surrounding fluid, $\varepsilon_{f}$, is generally assumed to be close to unity.

\section{RESULTS}

To verify the simulation methodology, results from random particle arrangement were compared with those reported by Beetstra (2005). Figure-2(a) and (b) shows a comparison between the calculated drag forces from our simulations to those reported by Beetstra (2005) for low (0.2) and high (21) particle Reynolds numbers respectively. Figure-2 also shows comparison between the calculated normalized drag and empirical drag models such as the Ergun and Wen-Yu models (Wen and $\mathrm{Yu}$, 1966). Except for very low overall voidage (close to minimum fluidization voidage), the simulations closely agree with those from Beestra (2005). The discrepancy at low with those of Beetstra (2005) could not be explained. At very high overall voidages (close to unity), the calculated drag forces from both this study and Beetstra (2005) reasonably agreed with that calculated from the Wen-Yu model. At low voidages close to minimum fluidizing voidages simulations from this study were closer to that of the Ergun model (Ergun, 1952). These observations were consistent for results from even higher Reynolds number runs which are not reported here. Furthermore, the drag forces from the LB simulations were considerable different from that from the empirical models, particularly at high volume fractions and particle Reynolds numbers. This observation was consistent with those of Hill et al. (2001) and Beetstra (2005).

\section{Effect of a cluster on gas-solid drag}

Simulations were carried out with particle configurations (as shown in Figure-1) which consisted of 64 particles forming a single cluster and 26 surrounding particles. In these configurations (first five configurations in Table-3), inter-particle distance between the particles in the cluster was varied from 0.9 to 0.52 ; with corresponding variation in cluster fractions (Table-3). However, particle Reynolds number and overall voidage in these configurations were kept constant at 420 and 0.985 respectively. The resulting drag force is shown in Figure-3.

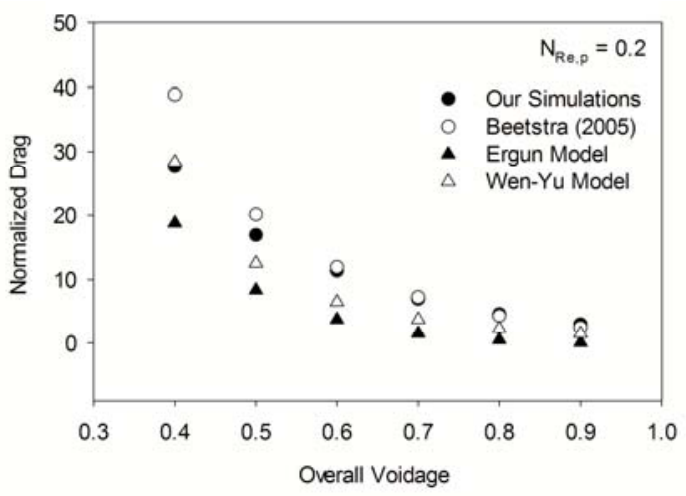

(a)

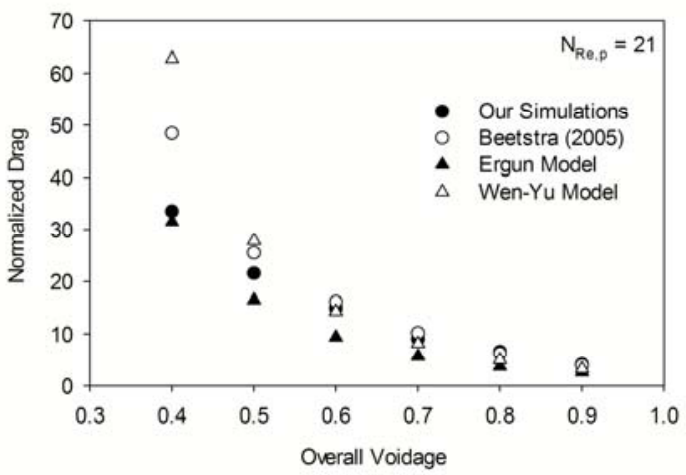

(b)

Figure 2: Normalized drag force Vs. Overall voidage (a) $\mathrm{N}_{\mathrm{Re}, \mathrm{p}}=0.2$ and (b) $\mathrm{N}_{\mathrm{Re}, \mathrm{p}}=21$

At higher voidages $(0.7$ to 0.97$)$ the calculated normalized drag force gradually declined with decrease in the cluster voidage. Further reduction in cluster voidage results into only marginal reduction in drag force. As the cluster voidage is increased, the calculated normalized drag force approaches that for the random configuration with the same overall voidage and particle Reynolds number. Based on these results, the cluster voidage of 0.7 was used in all subsequent simulations.

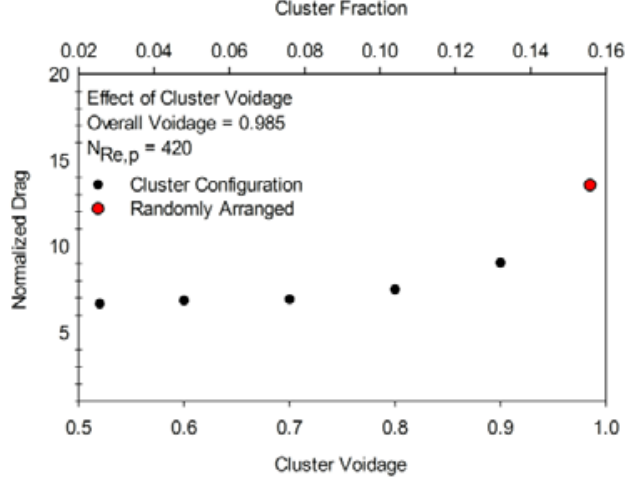

Figure 3: Normalized drag force Vs. Cluster voidage (Overall voidage $=0.985$ and $\mathrm{N}_{\mathrm{Re}, \mathrm{p}}=420$ )

Figure 4 shows a comparison between calculated drag forces from (i) random configuration and (ii) cluster configuration of 90 particles with a constant cluster 
voidage equal to 0.7 . The overall voidage in these simulations was constant at 0.985 , whilst the particle Reynolds number varied from 21 to 1049 . The simulation results show that the drag for cluster configuration was significantly lower than that for a randomly arranged particle configuration for the entire range of particle Reynolds numbers. Moreover, the difference between the two curves widened with increase in particle Reynolds number. These observations of drag reduction due to a cluster was consistent with the energy minimization multiscale (EMMS) concept (Li and Kwauk, 1994); which says that the formation of clusters causes less resistance to the flow of the fluid, and as a result it decreases the effective drag force. The simulation results provide a basis for quantifying such a reduction.

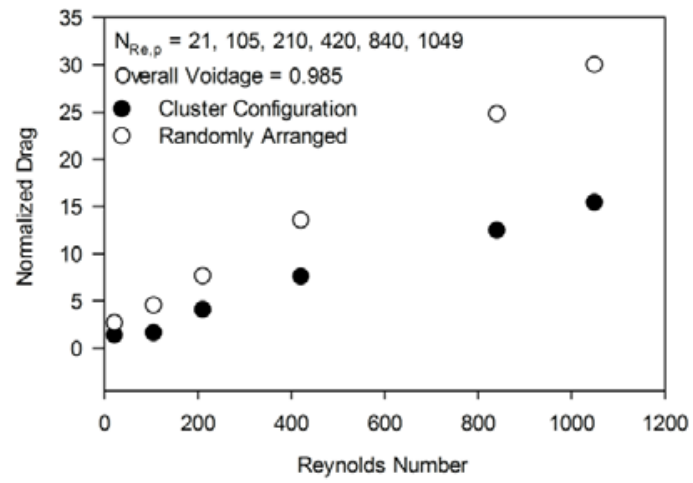

Figure 4: Normalized drag force Vs. Particle Reynolds number $($ Overall voidage $=0.985)$

\section{Effect of a cluster fraction}

Simulations were carried out with increasing number of particles in a cluster, from 64 to 1331, with a constant number of surrounding particles. Six particle configurations (see configurations 3 and 6-10, Table-3) were considered. Each configuration had different overall voidage but the same cluster voidage of 0.7 . Such an arrangement resulted in a variation in cluster fraction from 0.0502 to 0.7417 . Figure 5 shows the effect of overall voidage and cluster fraction on the calculated normalized drag force. It can be seen that as the voidage was increased, the solid phase resistance decreased which lowered the normalised drag. However, beyond an overall voidage of 0.92 , an increase in the normalized drag force was observed. This is due to the cluster behaviour transiting into randomly arranged particles at very high voidage, resulting in the normalized drag force approaching the value calculated for randomly arranged particles at the maximum voidage. Below overall voidage of 0.92 , decrease in the overall voidage with increase in the cluster fraction gave steep increase in the drag force which then seems to be approaching the value of the drag force for randomly arranged particles with approximately 0.7 overall voidage.

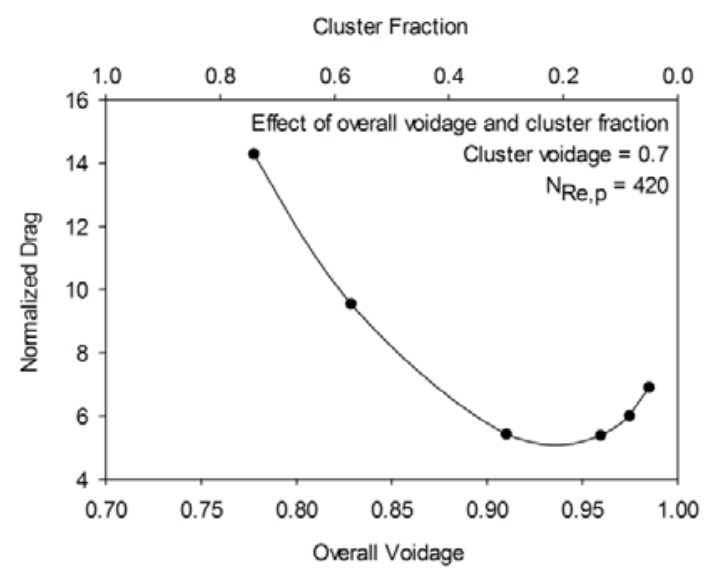

Figure 5: Effect of cluster fraction on calculated normalized drag forces.

Simulations were also carried out at different particle Reynolds numbers to study the effect of cluster fraction at constant cluster voidage. Figure 6 shows the calculated normalized drag calculated from simulations of six particle configurations (5-10, Table-3) with different particle Reynolds numbers ranging from 21 - 1049. For all simulations, a minimum drag force in the range 0.9-0.95 overall voidage was obtained, The minimum value of the drag force was found to increase with increased particle Reynolds numbers.

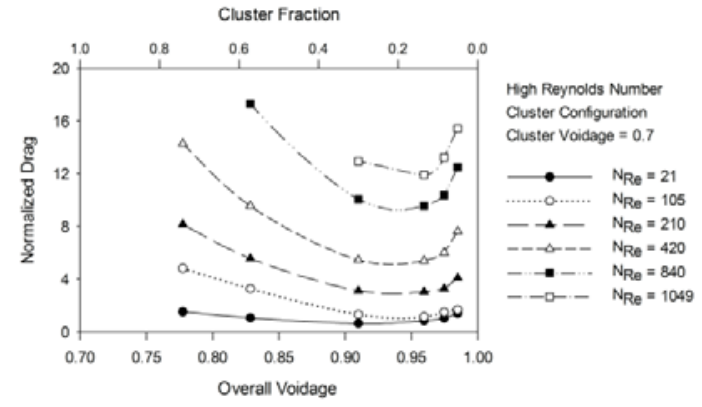

Figure 6: Effect of a cluster on drag at different flow conditions.

The simulations carried out as part of this study depended i.e. (i) overall voidage, (ii) particle Reynolds number, (iii) cluster fraction, and (iv) cluster voidage. Overall voidage, cluster voidage and cluster fraction are related by eq (14). Hence, flow can be defined by knowledge of three parameters. Information on the cluster voidage at given flow conditions is not available either experimentally or numerically. In the present study the cluster voidage was assumed to be constant. Work on predicting the cluster formation and cluster configuration for a given set of flow conditions is currently under progress.

\section{CONCLUSION}

Gas-particle flows with the particles arranged in the cluster configurations were simulated using LBM to investigate the effect of a single cluster and its properties on the gas-solid drag force over a wide range of voidages and particle Reynolds numbers.

When the calculated drag forces from the simulations with particles in the cluster configuration were compared with 
that for randomly arranged particles under the same flow conditions, the cluster configuration gave considerably lower effective gas-solid drag force. The reduction in the calculated drag force due to the cluster was observed to be larger at higher particle Reynolds numbers. The simulations with different particle configurations with varying inter-particle distances between particles in the cluster and a constant overall voidage showed that a major drag reduction happened for the cluster voidage higher than 0.7 , whereas a minor decrease in the drag force was observed for the cluster voidage less than 0.7 . When simulations were conducted a constant cluster voidage of 0.7 , the drag decreased with decrease in the overall voidage from the maximum voidage to approximately 0.92 . However, further decrease in the overall voidage resulted in steep increase in the calculated drag force. The findings reported here are important in formulating improved drag correlations that can be used in CFD simulations that solve the average two-fluid equations.

\section{ACKNOWLEDGEMENT}

Authors would like to acknowledge Prof. Anthony J. C. Ladd for SUSP3D code and iVEC for providing access to supercomputing facilities. This work was part of ARC funded research grant LP110100717.

\section{REFERENCES}

GIDASPOW, D. (1994) Multiphase flow and fluidization: continuum and kinetic theory descriptions, Academic Press.

ERGUN, S. (1952) Fluid flow through packed columns. Chemical Engineering Progress, 48, 89-94.

WEN, C. Y. \& YU, Y. H. (1966) Mechanics of fluidization. Chemical Engineering Progress Symposium Series.

ANDREWS IV, A.T., LOEZOS, P.N., SUNDARESAN, S., (2005), "Coarse-grid simulation of gas-particle flows in vertical risers", Industrial \& Engineering Chemical Research 44, 6022-6037.

LI, J., and KAWAUK, M., (1994), "Particle-fluid twophase flow: The energy-minimization multi-scale method”, Metallurgical Industry Press.

BENYAHIA, S., (2009), "On the Effect of Subgrid Drag Closures". Industrial \& Engineering Chemistry Research 49, 5122-5131.

SHAH, M.T., UTIKAR, R.P., TADE, M.O., EVANS, G., PAREEK, V.K., (2011), "Simulation of gas-solid flows in riser using energy minimization multiscale model: Effect of cluster diameter correlation" Chemical Engineering Science, 66, 3391-3300.

HILL, R.J., KOCH, D.L., LADD, A.J.C., (2001a),. "The first effects of fluid inertia on flows in ordered and random arrays of spheres". Journal of Fluid Mechanics, 448, 213241.

HILL, R.J., KOCH, D.L., LADD, A.J.C., (2001b), "Moderate-Reynolds-number flows in ordered and random arrays of spheres", Journal of Fluid Mechanics, 448, 243278.

VAN DER HOEF, M.A., VAN SINT ANNALAND, M., DEEN, N.G., KUIPERS, J.A.M., (2008), "Numerical simulation of dense gas-solid fluidized beds: a multiscale modeling strategy", Annual Review of Fluid Mechanics 70, 40-70.

BEETSTRA, R., VAN DER HOEF, M. A. , KUIPERS, J. A. M. (2006), "A lattice-Boltzmann simulation study of the drag coefficient of clusters of spheres", Computers \& Fluids, 35, 966-970.

ZHANG, Y., GE, W., WEI WANG, X., HE YANG, C. (2011), "Validation of EMMS-based drag model using lattice Boltzmann simulations on GPUs", Particuology, 9, 365-373.

LADD, A. J. C. (1994), "Numerical simulations of particulate suspensions via a discretized Boltzmann equation. Part 1. Numerical results", Journal of Fluid Mechanics, 271, 285-309.

LADD, A. J. C. (1994), "Numerical simulations of particulate suspensions via a discretized Boltzmann equation. Part 2 Numerical results", Journal of Fluid Mechanics, 271, 311-339.

BHATNAGAR, P. L., GROSS, E. P. , KROOK, M. (1954), "A model for collision processes in gases. I. Small amplitude processes in charged and neutral onecomponent systems”, Physical Review, 94, 511.

BEETSTRA, R. (2005), "Drag force in random arrays of mono-and bi-disperse spheres", $\mathrm{PhD}$, University of Twente. 